ISSN 1112-9867

\title{
LINKAGE BETWEEN RINGGIT EXCHANGE RATE AND U.S DOLLAR
}

\author{
N. N. Idalisa ${ }^{1 *}$, M. Tismazammi ${ }^{2}$ and M. A. N. Azwani ${ }^{2}$ \\ ${ }^{1}$ Faculty Computer and Mathematical Sciences, Universiti Teknologi MARA, Terengganu \\ Malaysia \\ ${ }^{2}$ Faculty of Business and Administration, Universiti Teknologi MARA, Dungun, Terengganu, \\ Malaysia
}

Published online: 17 October 2017

\begin{abstract}
In Malaysia, the movement of its currency rate i.e. Ringgit as against to US dollar are unpredictable and unregulated. Therefore, this causes some difficulty to appreciate the movement of macroeconomic analysis and market surveillance purposes. In 1965, Chaos theory was introduced, and it focused on behaviour and condition of dynamical systems. Chaotic behaviour involves the sequence of produced data in forecasting future performance, which is the cause of gaining unpredictable and random data. Nonetheless, while it is possible to present data by fixed order and mathematical formula, it is also possible that applications from various fields may contribute as a factor that affecting the movement of currency. This article investigates the behaviour of Ringgit movement as against to US dollar and result shows that Ringgit possess a chaotic behaviour in its currency movement.
\end{abstract}

Keywords: chaos theory; chaotic behaviour; non-linear model; fixed exchange rate; floating exchange rate.

Author Correspondence, e-mail: nuridalisa@tganu.uitm.edu.my

doi: http://dx.doi.org/10.4314/jfas.v9i5s.54 


\section{INTRODUCTION}

Translating the real problems into a mathematical form is the essential part of modelling. The process of modelling could involve a long discussion to clarify the problem, variables identification, estimation, approximation and many more activities which incurred more money and time. Mathematical modelling is conducted to imitate a real system and could be manipulated by researchers to solve real problems. Various models can be produced to design one particular real system and similarly, one model could be applied to different real problems. As a consequence, one model could perform better in one application but having poor performance in another application. Generally, the validity and success of a model depends on how it can be easily implemented and could provide accurate predictions.

Building a model is not only based on dynamical data available, but also requires extra information. Additionally, one of the challenges in composing a model is to acquire good and sufficient data [1]. Further challenges, inter alia may derive from the presence of noise, poor choice in observation, poor frequency content exist in the data, and limited amplitude excursion. Regardless of the linear behaviour used in modelling, many real systems appear to have non-linear behaviour. Unfortunately, majority of the nonlinear systems cannot be settled analytically hence no rigorous solution can be found. Therefore, the nonlinear model has been regularly transformed into the linear model by linearization process in order to get the approximation of solutions. However, the effect from linearization can hide some interesting behaviour of the exact solutions for example, chaos. Chaos has been defined as a non-linear behaviour arises between the periodic and random [2]. In Mathematics, chaos has been referred as the study of behaviour and condition of dynamical systems that appear to be very sensitive to the initial conditions

Chaos is divided into deterministic chaos and nondeterministic chaos; which the former represents the chaotic motion of non-linear systems whose dynamic laws uniquely determine the evolution of the system's state based on the previous evolution [18]. Examples of discipline that have deterministic chaotic behaviour in its application are engineering, electronics, geophysical applications, macroeconomic, monetary and financial markets series; for the purpose of controlling or forecasting.

On one hand, the time series data of ecnomic movement may be seen as unpredictable and 
unregulated. On the other hand, many research have proven that chaos exists in economy [11]. The emergence of advanced technology and computing tools make it easier to conduct a study of chaos. By using chaos theory, one could benefit from its applicability to highly nonlinear system where the theory considers every crucial system dynamics, uncovering the system information and relationships without further violating latent laws or dynamics [2]. In particular, the effect of chaos theory on economy is that it provides a clear explanation of the business cycle hence allowing the control of price level and improving the policies [14].

The problem stated refers to the daily fluctuation of currency movement which is due to uncertainty level in economy. Moreover, it is hard to predict the pattern of the data. The data is based on good condition or bad condition that occurs in Malaysia's currency. Previously, stochastic model is widely used by researchers to predict the price's pattern of commodity markets. However, if the data is chaos in behaviour, thus this model will obtain unsatisfied result. Hence, this study is to check the evidence or existing of chaotic behaviour in Malaysia's currency.

Due to the uncertain level of the economy, the movement of currency varies every day hence it is difficult to forecast the pattern of data. Data are based on the condition that affecting the Malaysia's currency; either good or bad condition. Stochastic model had been widely used by researchers to forecast the pattern of commodity market price, but it may provide an unsatisfied result for data that have chaotic behaviour. Therefore, this study aims to examine whether there is any evidence of chaotic behaviour exists in Malaysia's currency.

\section{LITERATURE REVIEW}

The standard national currency in Malaysia is known as the Malaysian Ringgit (RM), previously known as Malaysian Dollar (M\$). RM1 is equivalent to 100 cents in Malaysia. Locally, Malaysian Ringgit is symbolized as RM, but internationally is symbolized as MYR. There are various exchange rate policies that have been applied in Malaysia, which depending on the invisible hands (demand and supply) of foreign exchange market. Bretton Woods system is the current exchange rate system used in global economy that has been developed since 1958 [7]. In general, there are two types of exchange rate system, which the first type is known as fixed system and the other is floating system. A floating exchange rate system 
means that the rate varies all the time and has volatility, while a fixed exchange rate gives an opposite effect.

Trading in foreign exchange markets involves the conversion of currencies among countries, which allowing easy money flows. Many countries fixed their currencies in US dollar (USD) because most exchange rate trades involve in USD [9]. Consequently, it is very difficult to forecast the changes of foreign exchange rate in a particular country because of two different currencies involve are often fluctuate.

In the periods of 1997-1998, the Asian financial crisis that started in Thailand had affected vastly to other Asian countries including Malaysia. As a result from that crisis, many theories were discussed and proposed, which inter alia contained the causes of financial crisis, how policy responses to it, the impact of it, and solutions to control it [8] [12] [19]. Throughout the years, many have learned that the movement of currency is influenced by various matters. It is unfortunate that MYR is one of the worst performing currency over the past few years and its rate has been declining as against USD. The deterioration rate of MYR may be caused by 1) the impact of likely Fed rate hike; 2) the potential portfolio flows; and/or 3) the response of Bank Negara Malaysia (Malaysia National Bank) upon its weak currency [20]. Furthermore, other matters such as the significant decline in commodity prices and domestic politic issue i.e. $1 \mathrm{MDB}$ contributed to the drop of MYR rate.

Some analysis such as Lahmiri [13] has attempted to draw fine distinction of chaos in exchange rate. The scholar examined of chaos in Moroccan exchange rate versus British Pound. The results in short term and long term dynamic Moroccan exchange rate give a different chaotic pattern depending on the currency. Instantly, between short term and long term, the currency chaos exists in dynamic short term as compared to long term where there is no existence relationship.

There are many statistical methods available to analyse the movement of currency suggested by previous literatures. However, these methods are complex, random, and give unpredictable result. To name a few, the tests, which have been suggested to identify chaos in time series are entropy and BDS test, Lyapunov exponent, dynamical tool, the correlation dimension, the capacity dimension [10] [16] and 0-1 test by Gottwald and Melbourne [4].

The correlation dimension test provides the simplicity of the computation involved. This test 
nevertheless only provides the necessary, no adequate conditions to test the existence of chaos [10]. Therefore, this test depends on a very large and clean data set hence a few thousand observations may not be sufficient.

The test that computes Lyapunov exponent $\lambda$ is called the Lyapunov exponent test. The largest positive value of $\lambda$ will indicate chaos and vice versa, which this value may be calculated by experimenting the data without equations of a particular system. It also measures the rate of separation between two initially close trajectories [10]. To name a few methods that can estimate the value of $\lambda$ are by using the phase space reconstruction method, by approximating the linearization of the evolution operator, the tangent space method, and the direct method [3]. This test has been designed for 1) a large amount of data with at least 10,$000 ; 2$ ) a data that is not polluted by noise; and 3) a data that contain appropriate amount of aggregation because without aggregation means initially there has been no chaos [16].

Another most frequent used test of chaos is the BDS test that is not direct, but is used for non-linearity. The classic requirements in testing methods are the need for a very large and good quality data that is free from noise. However in reality, it is hard to acquire such data with such requirements. Due to this difficulty, many researchers have creatively discovered alternative methods to solve this issue. For example, Gottwald and Melboune [4] had proposed 0-1 test method, and Gottwald and Melbourne [3] had compared the Lyaponuv and $0-1$ chaotic tests. It was found that $0-1$ test is better than Lyapunov test. The reasons for such findings are: 1) the 0-1 test works directly on data and does not require phase space reconstruction; 2) the $0-1$ test is a binary test that provides a definite outcome; and 3) the $0-1$ test is able to tolerate moderate amount of noise that appear in data series.

The Visual Recurrence Analysis that is based on topological approach is another test of chaos [10]. In 1991, Aczel and Josephy published a paper in which they described the use of chaotic behaviour. A research of Singapore and other Europeans countries had been conducted. The results for Singapore dollar have smaller correlation compared others European countries. This means using this test, European countries has a significant correlation dimensions. 


\section{METHOD AND MATERIAL}

This paper will implementing the chaotic binary test or also known as 0-1 test [4]. The purpose of this method is to quantify the periodic and non-periodic attractors for chaos detection. The 0-1 test combines the spectral and statistical properties of the system [17]. This method can be summarized by Fig.1. 


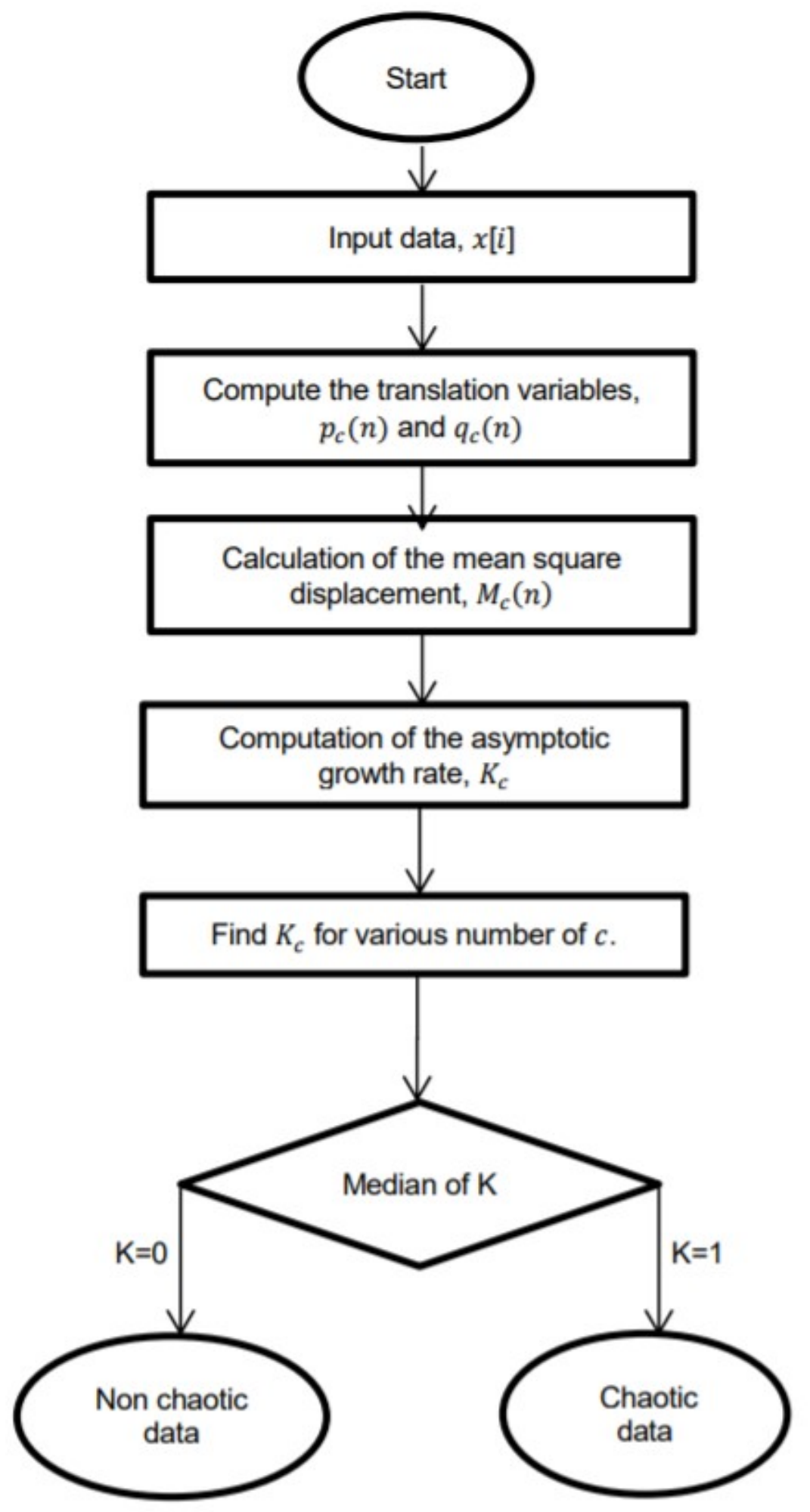

Fig.1. 0-1 test method

Suppose $x(i)$ is the observable data with respect to time. The first step in the $0-1$ test is to 
transform the observable data to variable $p$ and $q$. In order to demonstrate different types of dynamic of the system, the variable $p$ and $q$ are calculated by several number of parameter $c \in(0, \pi)$. Therefore, it is preferable to use the notation $p_{c}(n)$ and $q_{c}(n)$ with the following definition:

$$
p_{c}(n)=\sum_{i=1}^{n} x(i) \cos (i c) \quad, \quad q_{c}(n)=\sum_{i=1}^{n} x(i) \sin (i c)
$$

Notice that Eq. (1) resembles the Fourier transform for a chosen frequency. The full trajectory in the p-q plane will indicate the chaotic motion [5]. However, in practice to find the complete trajectory will require more computation time. Furthermore, the dynamic of the trajectory in p-q plane do not only depict the regular (periodic or quasiperiodic dynamics) but also chaotic dynamic that is tends to exhibit the two-dimensional Brownian motion with zero drift and hence evolve diffusively [4]. These dynamics could be conveniently distinguished by using mean square displacement (MSD) that can be computed as follows:

$$
M_{c}(n)=\lim _{N \rightarrow \infty} \frac{1}{N} \sum_{i=1}^{N}\left[p_{c}(i+n)-p_{c}(i)\right]^{2}+\left[q_{c}(i+n)-q_{c}(i)\right]^{2}
$$

However, the computation in Eq. (2) leads to another problem. The problem occurs in the infinite limit that enters its definition. In practice MSD is calculated for the time lags, $N / 100 \leq n \leq N / 10$ (Syta, et. Al, 2014). It is obvious to see that the displacement of the trajectories traveled in $\mathrm{p}-\mathrm{q}$ plane is varies for different time lag. The displacement is either bounded for regular dynamics or unbounded for chaotic dynamics. The linear growth of MSD with time indicates the diffusion movement and thus signals the chaotic dynamical system.

The asymptotic growth rate of MSD can be quantify by the value K using two methods; $K$ regression or correlation method. The regression method to calculate $\mathrm{K}$ by the following formula

$$
K_{c}=\lim _{n \rightarrow \infty} \frac{\log M_{c}(n)}{\log n}
$$

According to [4], the value $K_{c}$ can be approximated through minimizing the absolute deviation. This can be done by fitting a straight line to the graph of $\log M_{c}(n)$ versus $\log n$. 
The parameter $K_{c}$ must be computed for several values of $\mathrm{c}$ and the median of the corresponding value is the final indicator of the chaos behavior. If the median is approaching 1 then the chaotic level is high while nearly approach to 0 then the level of chaotic will be low. However, if the median is negative, the chaotic behaviour is not exist. Table 1 summarized the whole discussion on the dynamics happen in a time series from the $0-1$ test.

Table 1. Scenario of $0-1$ test

\begin{tabular}{llll}
\hline $\begin{array}{l}\text { Underlying } \\
\text { dynamics }\end{array}$ & Dynamics of $p(n)$ & $M(n)$ & $K$ \\
\hline Regular & Bounded $q(n)$ & bounded & 0 \\
\hline Chaotic & Diffusive & linear & 1 \\
\hline
\end{tabular}

This paper is considering the daily data of currency exchange of Malaysia Ringgit (MYR) to US Dollar (USD) from 2nd January 2010 until 7th December 2015. The data selection or rates was collected from the Interbank Foreign Exchange Market at Exchange Rates-Bank Negara Malaysia.

\section{ANALYSIS AND RESULTS}

Fig. 2 illustrates the asymptotic growth rate of $K_{c}$ for different value of parameter c. From the graphs, it is clear that value $K_{c}$ is approaching 1 for most parameter c. The median of $\mathrm{K}$ is $K_{c}=1.045110649 \approx 1$ 


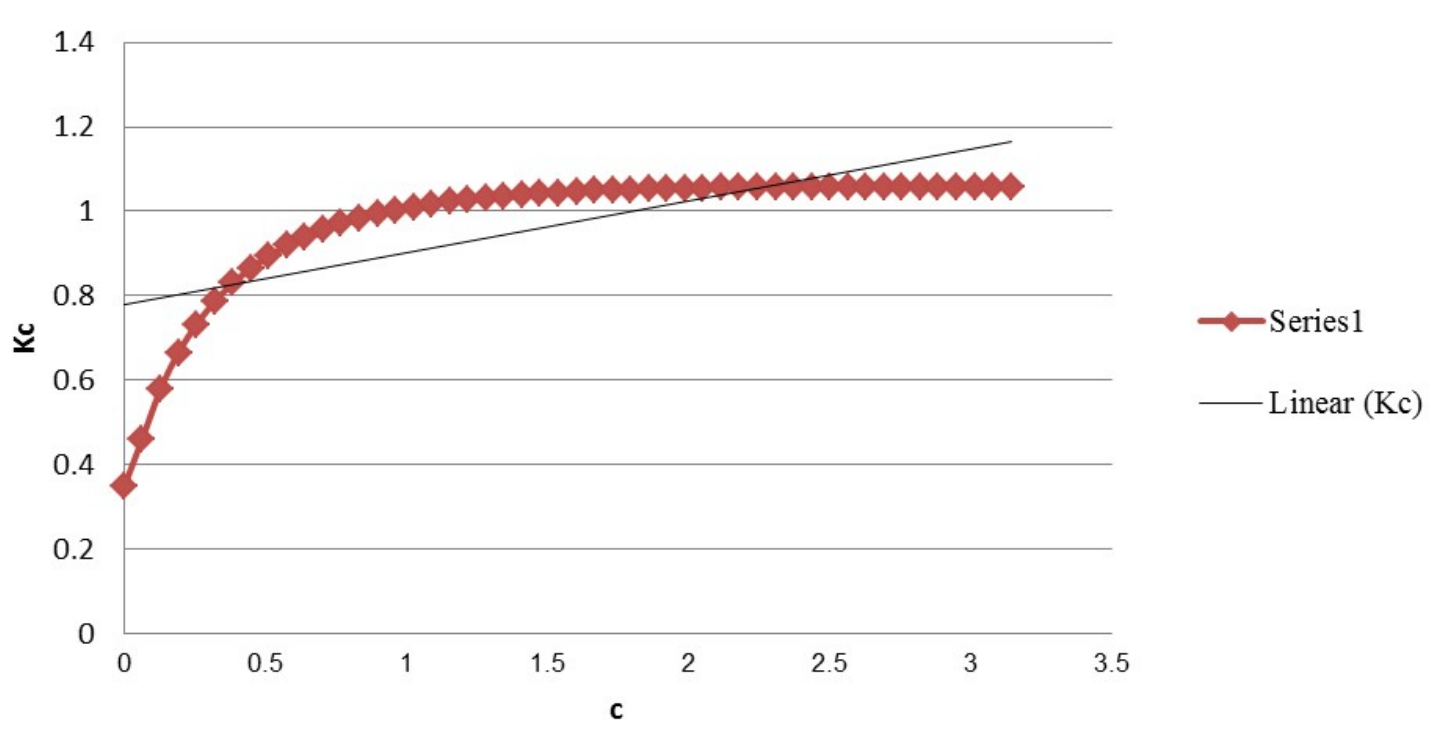

Fig.2. Asymptotic Growth Rate using regression method.
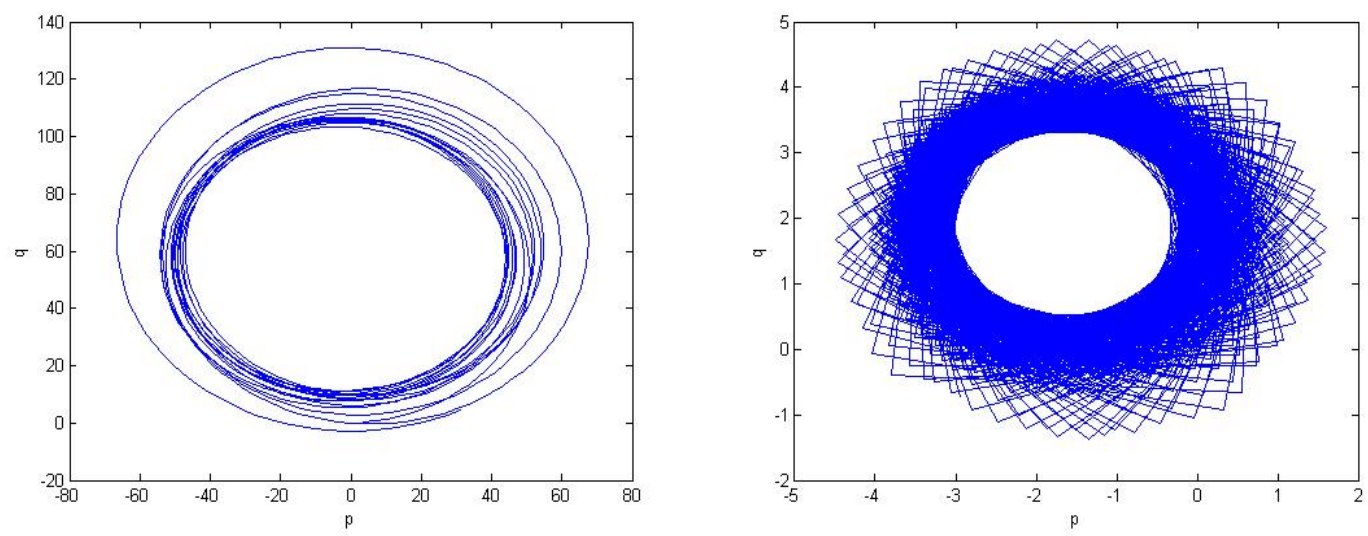

Fig.3. Plot $p$ versus $q$. Left: Regular dynamics at $\mathrm{c}=0.0641141357875468$; Right: Chaotic dynamics at $\mathrm{c}=1.60285339468867$. 

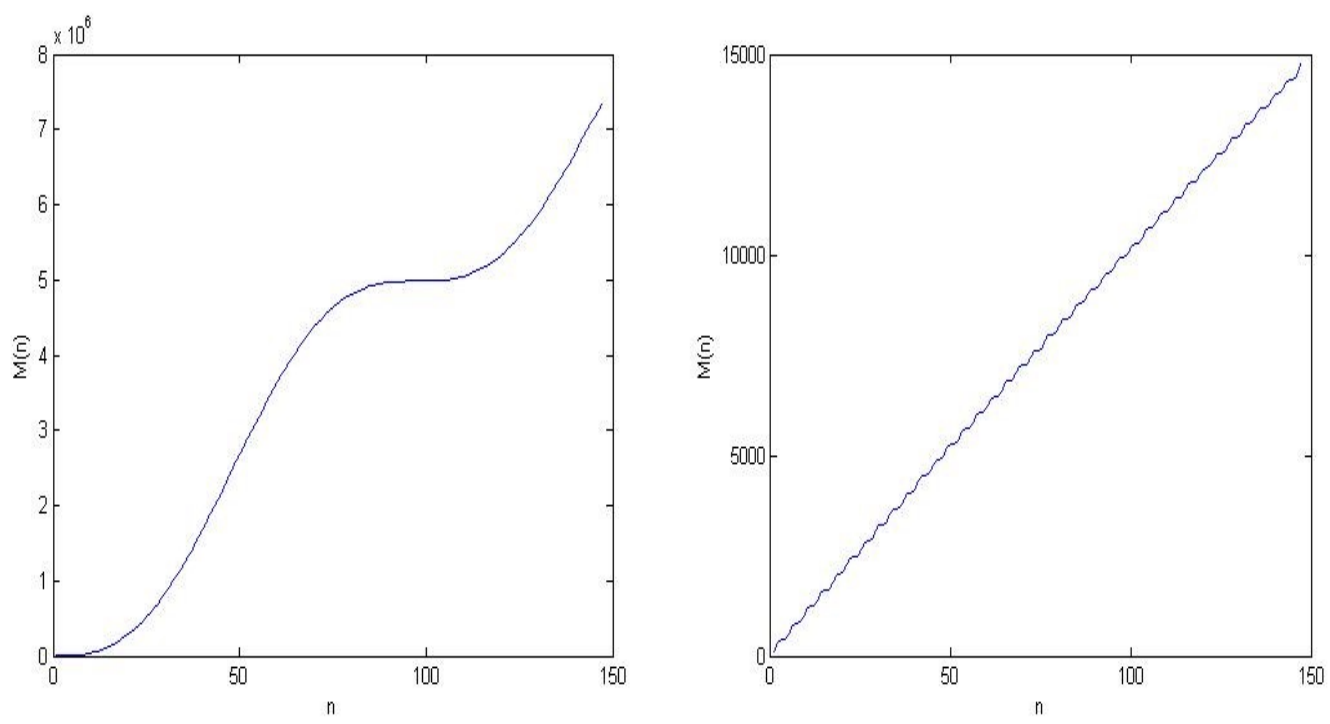

Fig.4. Plot MSD versus time lag n. Left: growth of MSD at c=0.0641141357875468; Right: growth of MSD at $\mathrm{c}=1.60285339468867$ linearly in time

\section{DISCUSSION}

Many have now shown their interest in the movement of currency. Being able to forecast future movement of currency in the exchange rate market will help the exchange rate traders to generate more income in trading. It is well noted that the movement of currency can be randomized or possessed certain pattern, which may able to reveal some information for future forecasting of its movement for the purpose of macroeconomic analysis and market surveillance. By holding to this notion, a 0-1 test had been conducted on the movement of MYR as against to USD and the result shows that the movement has chaotic behaviour.

Figures 3 and 4 shows that the dynamics in the p-q plane could exhibit chaos or regular chaos. There will be a collection of $p$ and $q$ with variation dynamics since the value of $p$ and $q$ are obtained from different value of $c$. Therefore, this indicates that the selection of value $c$ is crucial to determine the chaotic behaviour by using 0-1 test. A wrong selection of $c$ can lead to a false conclusion [5]. Thus, it is important to employ several values of $c$ in the calculation as one may not know exactly the correct value of $c$. It is common that the amount parameter $c$ should be at least 100 [3] and the outcome could be found by considering all values of $c$ when calculating the median of $K$. 
The median is estimated to be $K_{c}=1.045110649 \approx 1$ for the purpose of this project. It means that most calculation of $K$ that involved all values of $c$ resulted in a value that closes to 1 . For examples, the value $k=0.461343418308673$ leads to $c=0.0641141357875468$ and $k=1.04624484613573$ leads to $c=1.60285339468867$. Both Figures 3 and 4 make a comparison on the dynamics of currency data in p-q plane and the movement of MSD over time in two values of $\mathrm{c}$ that depicted opposite dynamic behaviour, respectively. Alternatively, the estimation of value $K$ that closes to 1 means that MSD is linked linearly depending on time, which also means the extent of currency data movement in specific time lag.

It is the nature of chaos system to be highly sensitive towards the initial condition. Even though it was fairly difficult to make forecasting based on chaos system, many have attempted to make it achievable. One of those exceptional researches is Ola [15] whom had applied the local polynomial model to predict the stock returns in Tehran and was able to show the existence of chaotic behaviour [6].

As aforementioned, there are various factors that may influence the chaotic behaviour, which its definition has been accepted as the sequence of produced data in forecasting future performance. Although the sequence of produced data in future performance can produce unregulated and random data, it is possible to be exemplified by fixed order and Mathematical formula. Moreover, the Chaos theory does not consider the market history and some extra information or issues, which may affect the movement of currency.

\section{CONCLUSIONS AND RECOMMENDATIONS}

The ability to predict the currency movement is important to many entities including the traders, investors, government and so on. This is because the prediction of the currency movement can be a guideline for strategic planning in order to minimize risks and maximize returns. Although there are numerous methods available to forecast the currency movement, none would give the precise result. This paper has discovered that the movement of ringgit poses chaos behaviour through the result from 0-1 test. Even though the chaotic behaviour does not directly produce equations to develop a model, it extracts valuable information involving all system dynamics. Further analysis is required to produce the mathematical 
modelling that can represent the currency movement and also to study the stability of the model. Nevertheless chaotic behaviour would give some hint on this and if using appropriate methods, a short-term prediction can be made.

\section{REFERENCES}

[1] Luis A A, Letellier C. Mathematical Problems in Engineering, 2009, 1-35, doi: $10.1155 / 2009 / 238960$

[2] Frazier C, Kockelman K. C. Transportation Research Record, 2004, 1897(1), 9-17. doi:10.3141/1897-02

[ 3] Gottwald G A Melbourne I. Physica D: Nonlinear Phenomena, 2005, 212(1-2), 100-110, doi:10.1016/j.physd.2005.09.011

[4] Gottwald G A, Melbourne I. The 0 - 1 test for chaos: A review. In C. Skokos, G. A. Gottwall, \& J. Laskar (Eds.), Chaos detection and predictability. Springer Berlin, Heidelberg: Lecture Notes in Physics, 2016, pp. 221-247

[5] Bernardini D, Grzegorz L. An overview of 0-1 test for chaos. Journal of Brazilian Society of Mechanical Sciences and Engineering, 2016, 38(5):1433-1450

[6] Aczel A D, Josephy N H. The chaotic behavior of foreign exchange rates. The American Economist, 1991, 35(2):16-24

[7] Bordo M D, Humpage O F, and Schwartz A J U S. Intervention during the bretton woods era: 1962-1973. The National Bureau of Economics Research, 2011

[8] Choong F L, Meera A K. Managing foreign exchange risk: The Malaysian experience in the 1997 financial crisis. The Philippine Review of Economics, 2007, 44(1):187-216

[9] Das P P, Das D A. Comparison of recession during 2008 - 2009 and 2009-2013 through chaotic analysis of the foreign exchange rates. Journal of Contemporary Management, 2013, 2(4):100-112

[10] Faggini M. Chaos detection in economics: Metric versus topological tools. Munich Personal RePEc Archive, 2010, pp. 1-20

[11] Guegan D. Chaos in economics and finance. Annual Reviews in Control, 2009, 33(1): 89-93 
[12] Hasan Z. The 1997-98 financial crisis in Malaysia: Causes, response and results. Islamic Economic Studies, 2002, 10(2):45-53

[13] Lahmiri S. Investigating existence of chaos in short and long term dynamics of Moroccan exchange rates. Physica A: Statistical Mechanics and its Applications, 2017, 465, 655-661

[14] Nabi M, Tash S. A study on non-linear and chaotic behavior of Iran's economic growth. International Journal of Academic Reseach in Business and Social Sciences, 2012, 2(10): $373-382$

[15] Ola M R, Nooghabi M J, Rounaghi M M. Chaos process testing (using local polynomial approximation model) in predicting stock returns in Tehran stock exhange. Asian Journal of Research in Banking and Finance, 2014, 4(11):100-109

[16] Silva S D. Chaos and exchange rates. 2009

[17] Syta A, Litak G, Lenci S, and Scheffler M. Chaotic vibrations of the doffing system with fractional damping. Chaos: An Interdisciplinary Journal of Nonlinear Science, 2014, 24(1):1-6

[18] Vlad S, Pascu P, and Morariu N. Chaos models in economics. Journal of Computing, 2010, 2(1):79-83

[19] Wang Y, Yang W, Li K, and Hu F. Prediction and control of financial crisis based on chaotic system. In Proceedings of the $20102^{\text {nd }}$ IEEE International Conference on Information and Financial Engineering, 2010, pp. 865-869

[20] Devesh D, Lai L, and Lee E. Malaysia-keep calm and carry on. Standard Chartered Local Markets Alert, 2015

\section{How to cite this article:}

Idalisa N N, Tismazammi M, Azwani M A N. Linkage between ringgit exchange rate and u.s dollar. J. Fundam. Appl. Sci., 2017, 9(5S), 758-771. 